\title{
Recombinant human MDM2 oncoprotein shows sequence composition selectivity for binding to both RNA and DNA
}

\author{
CHRISTINE CHALLEN $^{1 *}$, JOHN J. ANDERSON ${ }^{1 *}$, ZOFIA M.A. CHRZANOWSKA-LIGHTOWLERS ${ }^{2}$, \\ ROBERT N. LIGHTOWLERS ${ }^{2}$ and JOHN LUNEC ${ }^{1}$ \\ ${ }^{1}$ Northern Institute for Cancer Research, ${ }^{2}$ Institute For Cell and Molecular Biosciences, \\ Newcastle University Medical School, Newcastle-upon-Tyne, NE2 4HH, UK
}

Received August 2, 2011; Accepted September 19, 2011

DOI: 10.3892/ijo.2011.1267

\begin{abstract}
MDM2 is a $90 \mathrm{kDa}$ nucleo-phosphoprotein that binds p53 and other proteins contributing to its oncogenic properties. Its structure includes an amino proximal p53 binding site, a central acidic domain and a carboxy region which incorporates Zinc and Ring Finger domains suggestive of nucleic acid binding or transcription factor function. It has previously been reported that a bacculovirus expressed MDM2 protein binds RNA in a sequence-specific manner through the Ring Finger domain, however, its ability to bind DNA has yet to be examined. We report here that a bacterially expressed human MDM2 protein binds both DNA as well as the previously defined RNA consensus sequence. DNA binding appears selective and involves the carboxy-terminal domain of the molecule. RNA binding is inhibited by an MDM2 specific antibody, which recognises an epitope within the carboxy region of the protein. Selection cloning and sequence analysis of MDM2 DNA binding sequences, unlike RNA binding sequences, revealed no obvious DNA binding consensus sequence, but preferential binding to oligopurine:pyrimidine-rich stretches. Our results suggest that the observed preferential DNA binding may occur through the Zinc Finger or in a charge-charge interaction through the Ring Finger, thereby implying potentially different mechanisms for DNA and RNA MDM2 binding.
\end{abstract}

\section{Introduction}

The $M D M 2$ oncogene was originally cloned from a spontaneously transformed BALB/c 3T3 mouse cell line in which it was found to be amplified and overexpressed $(1,2)$. p53 can induce the expression of MDM2 via transactivation of a p53 binding

Correspondence to: Professor John Lunec, Northern Institute for Cancer Research, Newcastle University Medical School, Newcastleupon Tyne, NE2 4HH, UK

E-mail: john.lunec@ncl.ac.uk

*Contributed equally

Key words: MDM2, DNA binding, RNA binding, RING Finger site located in intron 1 of the MDM2 gene, thereby activating an auto-regulatory feed-back loop (3-5). Complex-formation between these proteins targets p53 for ubiquitin-dependent proteolysis (6). MDM2-p53 complex formation also inhibits the ability of p53 to transcriptionally activate gene expression $(7,8)$. Since these early experiments, research has revealed multiple binding activities, suggesting that MDM2 may be a molecule capable of a variety of functions. Indeed studies have revealed that the $M D M 2$ gene encodes a variety of alternatively spliced mRNAs, some of which retain transforming activity despite the loss of sequences from the 5' end of the MDM2 gene that encodes the p53 binding site (9). Expression of alternatively spliced forms of MDM2 has been associated with advanced tumour stage and high histological grade in carcinoma of the urinary bladder and ovary. In addition to binding and regulating p53, MDM2 has been shown to bind other proteins which may modulate or contribute to its transforming/oncogenic properties. MDM2 has been demonstrated to interact with the human TATA binding protein (TBP) both in vitro and in vivo in the absence of p53. This suggests that MDM2 may have a role in the regulation of gene expression independent of p53. In addition, it has been reported that MDM2 binds and complexes with the retinoblastoma protein, inhibiting its growth regulatory function by relieving its suppression of E2F-1 transactivation function (10). MDM2 can also bind to the E2F1/DP1 transcription factors and stimulate transcription required for S-phase cell cycle progression (11). MDM2 has also been shown to interact with the L5 ribosomal protein associated with 5S ribosomal RNA, although the function of this complex is unknown (12). Recent reports show that MDM2 binds to $\mathrm{p} 73$, which has high structural homologies with p53. This binding complex formation again impairs the capacity of p73 to act as a transcriptional activator. Unlike p53, however, p73 is not destabilised by MDM2 (13).

Deletion mapping and sequence analysis have shown that the $90 \mathrm{kDa}$ MDM2 nuclear phosphoprotein contains a p53 binding site in the amino terminal region (codons 19-102), a putative nuclear localisation signal (codons 181-185), an acidic domain (codons 223-274), a central Zinc Finger motif (codons 305-322) and a carboxy-terminal RING Finger zinc binding motif (codons 438-478) suggesting a nucleic acid binding and/ or transcription factor function $(14,15)$. Bacculovirus expressed MDM2 has been shown to bind RNA specifically through the RING Finger domain of the protein. Furthermore, a single 
amino acid substitution in the RING Finger domain abrogates the specific RNA binding (16). Although the function(s) of the RING Finger are still being unravelled, it has been shown to be required for the degradation of p53 and down-regulation of intracellular MDM2 levels (13).

The RING Finger has been implicated in specific ubiquitination events (17), in particular the C-terminal RING Finger region of MDM2 has been suggested to play a role in the ubiquitinproteosome dependent degradation of p53 and of MDM2 itself (18). Honda et al have demonstrated that MDM2 functions as an E3 ubiquitin ligase for p53 in vitro (6). Tanimura et al have also shown that MDMX modulates ubiquitin-proteosome dependent proteolysis of MDM2 by interacting with its C-terminal RING Finger domain (19). In addition, the RING Finger may also be involved in protein-protein interactions and DNA-protein interactions that have as yet to be demonstrated. We have therefore examined both the DNA and RNA binding properties of bacterially expressed full length and C-terminal fragments of human MDM2. Gel mobility shift assays confirmed the ability of human MDM2 to bind both DNA and RNA. From our results, we propose, however, that DNA and RNA binding by MDM2 are mediated through different mechanisms.

\section{Materials and methods}

Cloning of MDM2 sequences from total cellular RNA and generation of MDM2 expression constructs. Methods for these are described fully in Anderson et al (20). Briefly total cellular RNA was extracted from sub-confluent mono-layers of $\mathrm{CaCo} 2-$ colonic carcinoma cells using the conventional manufacturer defined RNAzol based extraction protocols (Biogenesis Ltd., UK). Full and partial cDNA clones of MDM2 were generated by RT-PCR and the resulting constructs cloned, transformed and the inserts sequenced.

Selected inserts were used to generate MDM2 expression constructs which were then subsequently employed to make either a carboxy-terminal maltose binding protein (CT-MBP-MDM2) and/or polyhistidine tagged full length (MDM2-FL) protein for use in all binding reactions.

DNA binding studies: Selection of oligo-nucleotides corresponding to the putative MDM2 DNA binding site. A modification of the method of Pollock and Treisman was applied to amplify, enrich, select and identify sequence preferential DNA:protein interactions (21). This involved protein-DNA complex formation, column chromatography, PCR and sequencing. Briefly, selection began by synthesising a 26 base random oligonucleotide $(\mathrm{A} / \mathrm{G} / \mathrm{C} / \mathrm{T})_{26}$, flanked by sequences incorporating EcoR1 and Bam $\mathrm{H} 1$ restriction sites in regions corresponding or complementary to the primers PF (5'-GCTGCAGTTGCACTGAATTCGCCTC-3') and PmR (5'-GCTGTCGTACGCGACTTGACTGGAC-3'). This facilitated generation of a product corresponding to the $\mathrm{R} 76$ product described by Pollock and Treisman (21). A ${ }^{32} \mathrm{P}$ radiolabelled source of this product was prepared by PCR utilising PF and PmR primers in reactions in which an aliquot of the full R76 oligo-nucleotide served as the template. Reactions $(10 \mu \mathrm{l})$ were established incorporating 18 pmols of each primer, $200 \mu \mathrm{M}$ dATP, $5 \mu \mathrm{l}$ of $\left[\alpha^{-32} \mathrm{P}\right]$-dATP $(3000 \mathrm{Ci} / \mathrm{mmol}), 500 \mu \mathrm{M}$ each of dCTP, dGTP and dTTP, 10X standard reaction buffer, 0.5 units of Thermoprime plus DNA Taq polymerase and $0.5 \mathrm{ng}$ of the template. PCR cycling conditions were defined as: i) denaturation $93^{\circ} \mathrm{C}$ for $5 \mathrm{~min}$ in initial round, $1 \mathrm{~min}$ in all subsequent rounds. ii) Primer annealing $72^{\circ} \mathrm{C}$ for $1 \mathrm{~min}$ iii) primer extension at $72^{\circ} \mathrm{C}$ for $5 \mathrm{~min}$. Steps i) to iii) were repeated 14 further times. The radio labelled product was then purified by PAGE gel extraction and eluted in $400 \mu \mathrm{l}$ of TE. This was then used as the starting material in the first of further successive rounds of DNA binding site selection and chromatographic purification. Each round of selection involved amplification and labelling of the enriched R76 sub-species selected by the binding process. All binding reactions were performed in reaction volumes of $15 \mu 1$ and comprised $2.5 \mu \mathrm{g}$ of the CT-MBP-MDM2 protein, $3.2 \mu \mathrm{l}$ of $\mathrm{D}_{z}$ buffer $(5 \mathrm{mM}$ HEPES $\mathrm{pH} 7.9,20 \%$ glycerol, $2 \mathrm{mM}$ $\mathrm{Mg} \mathrm{Cl}_{2} 1 \mathrm{mM}$ DTT, $0.1 \mu \mathrm{M} \mathrm{ZnCl}_{2}, 0.2 \mathrm{mM}$ EDTA), $500 \mathrm{mM}$ $\mathrm{KCl}, 250 \mathrm{ng}$ Poly ( $\mathrm{dIdC}$ ) and $16 \mu \mathrm{g}$ of BSA. These reactions were first incubated on ice for $10 \mathrm{~min}$ prior to the addition of $2 \mu \mathrm{l}$ of the labelled R76n DNA from successive rounds of selection (R76 plus $n$ where $n=$ the number of rounds of selection after elution of nucleotides bound to MDM2 using amylose chromatography). These reactions were then incubated for a further $10 \mathrm{~min}$ at room temperature.

Chromatographic purification of DNA: protein complexes. Amylose column chromatography was used to purify DNA protein complexes at each round of selection. This was achieved by first adding $88 \mu 1$ of column buffer $(10 \mathrm{mM}$ phosphate, $0.5 \mathrm{M}$ $\mathrm{NaCl}_{2}$ and $1 \mathrm{mM} \mathrm{NaN}_{3}$ ) supplemented with Tween 20 to a final concentration of $0.25 \% \mathrm{v} / \mathrm{v}$. This mixture was subsequently loaded onto an equilibrated amylose resin purification column. The DNA protein binding reaction mixture was collected and re-loaded onto the column five times to ensure maximum binding to the amylose. After this procedure the column was washed with three column volumes of standard column buffer plus Tween to limit non-specific binding. Prior to elution, the bound complexes were further washed with five column volumes of standard column buffer in the absence of Tween. Complexes were eluted with a solution of standard column buffer augmented by the addition of $10 \mathrm{mM}$ maltose. Four $3 \mathrm{ml}$ fractions were collected on elution. Each was then ethanol precipitated, amplified by PCR and PAGE purified as described above. The process of successive rounds of amplification and selection of DNA:protein complexes by amylose column chromatography was repeated in all seven times to give 7 th round selected DNA.

Gel mobility shift analysis and further selection of DNA sequences binding to MDM2. DNA binding reactions were performed using a modification of the method described above using 7th round selected DNA and $10 \mathrm{mg}$ of full length-poly histidine tagged recombinant protein and incorporating excess Poly (dIdC) $(1000 \mu \mathrm{g})$ to restrict non-specific binding. Control reactions were also constructed in which the protein was omitted from the reaction mix. After completion of the reactions $2 \mathrm{ml}$ aliquots of loading buffer were added to test and control binding reactions, which were then analysed in parallel on continuous 5\% PAGE-TBE gel $\left(30 \mathrm{~V}-4 \mathrm{~h}\right.$ at $\left.4^{\circ} \mathrm{C}\right)$. Labelled DNA was then visualised by Phosphor-imaging and the mobility of the selected nucleotides compared in the presence and absence of recombinant MDM2 protein. The shifted band, corresponding to a 
DNA:MDM2 complex, was excised and eluted from a gel in $200 \mathrm{ml} \mathrm{TE}$ buffer. A sample of this extracted material was then amplified by PCR, utilising RF and PmR primers. Nine additional successive rounds of gel purification from mobility shift gels were performed to yield the final product $(7+10$ selected DNA).

Prior to cloning and sequencing the final oligo-nucleotide product, an additional series of experiments were performed to further ensure the specificity of the DNA:MDM2 binding. In these experiments micro-columns of Protein $\mathrm{G}$ were saturated with anti-MDM2 monoclonal antibodies (F4-14 and F2-2) which were used to absorb MDM2 protein from the full lengthMDM2 preparation used routinely in mobility shift assays (20). This absorption procedure was shown to successfully remove MDM2 protein from the preparation to a point where it proved undetectable using a combination of PAGE and silver staining. The absorbed material was then included as a control alongside unabsorbed material in a series of mobility shift assays. In addition a final negative control for any effects of the histidine tag was included in band shifts, which consisted of a preparation of poly-histidine-tagged cyclin D1 as an arbitrary non-DNA binding protein prepared in exactly the same manner as the fulllength MDM2 protein.

Cloning and sequencing of putative MDM2 DNA reactive sites. The enriched MDM2 DNA binding sequences (7th or 7+10) were PCR amplified using the PF and PmR primers as described previously. The resulting PCR products were cloned into the pCR $3.1^{\mathrm{TM}}$ vector using the TA cloning system (Invitrogen) following the manufacturers protocol. Constructs were then transformed into $E$. coli-INVaF, and established transformants subsequently grown on solid selective agar media incorporating, ampicillin and x-gal. Ampicillin resistant transformants were screened for inserts by PCR using SP6 and T7 primers (both sites being present in the pCR 3.1 cloning vector). Clones containing inserts of the appropriate size were PCR amplified using PF and PmR primers in the presence of ${ }^{32} \mathrm{P}-\mathrm{dATP}$ and the MDM2 binding activity of the products analysed in gel mobility shift assays against the full length MDM2 protein. The PCR products showing MDM2 binding activity were then directly sequenced. All reactions were performed in accordance with the manufacturers protocol (Amersham Sequenase 2.0) and the sequences with detectable binding activity comparatively aligned using Clustal W, Blast and DNAStar software.

RNA binding studies. Experiments were performed to establish and confirm both the RNA and p53 binding capacity of the bacterially expressed full length polyhistidine tagged MDM2 product employed in our binding studies.

Creation of an RNA probe corresponding to the reported MDM2 RNA binding site target sequence. A DNA oligo-nucleotide termed T7EBSp6 containing a 30 nucleotide sequence corresponding to the published RNA consensus sequence (16), together with flanking sequences incorporating T7 and SP6 promotor sites, was synthesised on an ABI 392 RNA/DNA synthesiser: (5'-TGTAATACGACTCACTATAGGGCGAAC CGCCGGAUCUUAAAUAAACUCCUGGUCUAGTTTCTA TAGTGTCACCTAAAT-3') (20). This synthetic oligonucleotide acted as the template to allow synthesis of a dsDNA intermediate which would facilitate in vitro transcription and ${ }^{32} \mathrm{P}-\mathrm{UTP}$ labelling of the desired RNA probe. The abovepurified dsDNA as well as a randomised control cassette of 50 nucleotides (N50) was used as a template to enable creation of the desired RNA probes [Ampliscribe SP6 in vitro transcription system, supplying $\alpha_{-}{ }^{32} \mathrm{P}-\mathrm{UTP}\left(400 \mathrm{Cimmol}^{-1}\right)$ as well as unlabelled CTP, GTP and ATP (Promega, USA, Ltd.)].

The resulting ribo-probes were treated with DNAse I for $30 \mathrm{~min}$ at $37^{\circ} \mathrm{C}$, phenol extracted and the RNA precipitated with 5.2 $\mathrm{M}$ ammonium acetate $\mathrm{pH} 4.8$ in ethanol (plus linear acrylamide as a carrier). Probes were pelleted at 14,000 g, washed with $70 \%$ ethanol, re-pelleted and re-suspended in 10-20 $\mu 1$ of DEPC treated water. Subsequently, the concentration and specific activity of the probe was determined prior to use in RNA:MDM2 binding assays as described below.

RNA:MDM2 binding assays. Full-length recombinant MDM2 protein (5 mg) was incubated together with $0.2-0.5 \mathrm{ng}$ $(100,000 \mathrm{cpm})$ of either of the above-labelled ribo-probes in a reaction volume of $15 \mathrm{ml}$. Sequentially the probe was diluted in the appropriate volume of binding buffer (0.2 mM EDTA, $2 \mathrm{mM}$ $\mathrm{MgCl}_{2}, 0.5 \mathrm{mM}$ DTT and 0.5 mM PMSF in PBS), heated to $70^{\circ} \mathrm{C}$, and cooled to $30^{\circ} \mathrm{C}$. After addition of the protein, binding reactions were allowed to proceed for $15 \mathrm{~min}$ at $23^{\circ} \mathrm{C}$, after which $20 \mathrm{U}$ of RNAse T1 was added to digest unprotected RNA. After $5 \mathrm{~min}$ at room temperature, $50 \mathrm{mg}(1 \mathrm{ml})$ of calcium heparin was added to reduce non-specific binding. Following a further $5 \mathrm{~min}$ period, reactions were chilled. The RNA moiety, was then covalently linked to the protein by UV irradiating the RNA:MDM2 complexes (720 sec in a standard Stratagene-Stratolinker). Finally the reaction mixture was incubated at $37^{\circ} \mathrm{C}$ with $20 \mathrm{U}$ of RNAse for $10 \mathrm{~min}$. At this point binding reactions were taken up in dissociation buffer and resolved by PAGE alongside probe treated in the same way, but in the absence of MDM2. Labelled products and their relative mobilities were visualised by Phosphorimaging. In a series of controls, the protein moiety was digested, after binding, with Proteinase $\mathrm{K}$ confirming the protein dependency of RNA binding. Controls lacking MDM2 protein in the initial incubation and post UV digestion phases were also included.

RNA:MDM2 super-shift assays. Super-shift assays were carried out to further test the MDM2 specificity of the binding activity inferred by the mobility shift assays. RNA binding assays were performed with the following modification: prior to incubating the protein with the radio-labelled oligo-nucleotide, a standard concentration of protein (5 $\mu \mathrm{g}$ full length recombinant MDM2) was incubated with increasing concentrations $(0.01$ to $1.8 \mu \mathrm{g})$ of one of the two immuno-affinity purified MDM2 specific monoclonal antibodies F4-14 and F2-2 (20). Controls were also established in which the protein was reacted with PBS alone or with an equivalent concentration of an antibody shown not to cross-react with recombinant MDM2. This antibody had been raised in our laboratories against cyclin D1 (22). Antibody: protein complexes were allowed to form over $2 \mathrm{~h}$ at $4^{\circ} \mathrm{C}$. Subsequently, binding reactions were analysed by non-denaturing Tris-Glycine (4-20\% T 2.6\% C) PAGE and discontinuous denaturing PAGE (23\% T plus $18 \%$ urea denaturant). Optimum results were obtained with conventional reducing SDS loading buffer supplemented with $54 \%$ urea. 
(A)

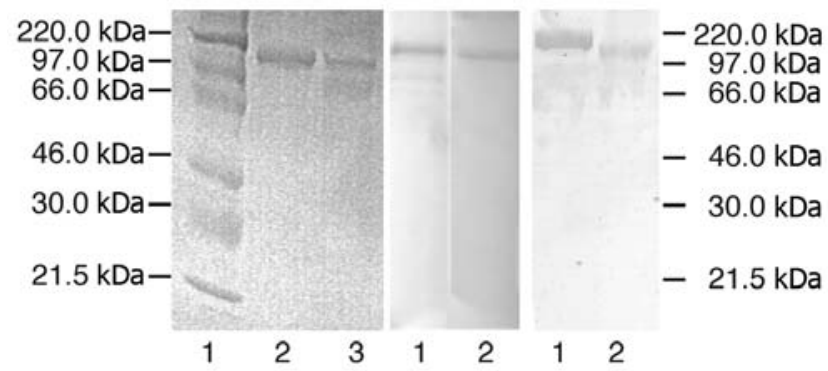

Figure 1. Coomassie stain of MBP-MDM2 and His tagged FL-MDM2 protein and Western blotting using monoclonal anti-MDM2 antibodies F2-2 and F4-14. Features shown are: (A) Coomassie blue stain of MBP-MDM2 and His-FL MDM2: Track 1, molecular weight markers; 2, MBP-MDM2; 3, His tagged FL-MDM2. (B) Western blotting of MBP-MDM2 and His-FL MDM2 probed with MAb F2-2. Track 1, MBP-MDM2; 2, His tagged FL-MDM2. (C) Western blotting of MBP-MDM2 and His-FL MDM2 probed with MAb F4-14. Track 1, MBP-MDM2; 2,His tagged FL-MDM2.

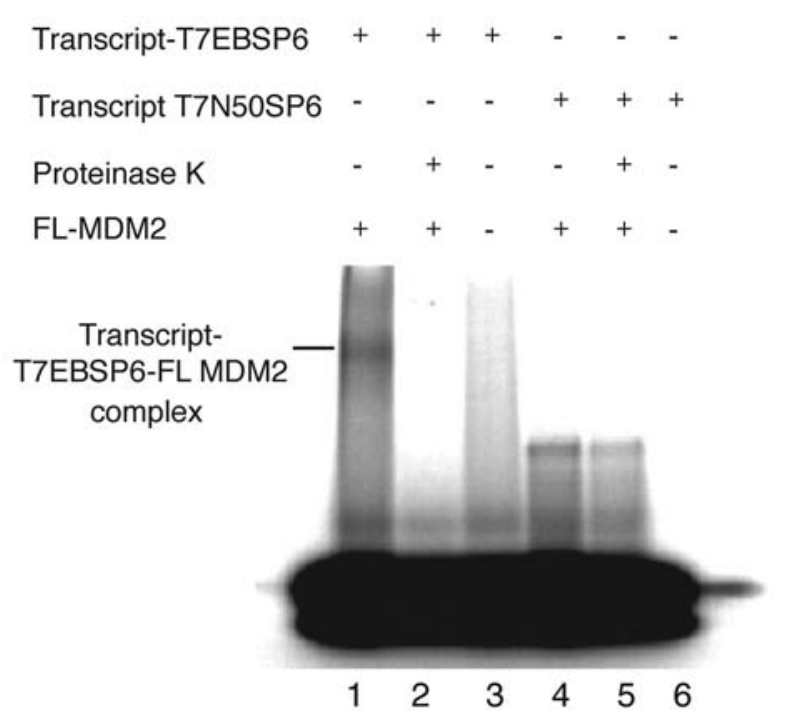

Figure 2. Demonstration of RNA binding of recombinant bacterial FL-MDM2 and obligate dependence of binding upon the presence of the protein. Features shown are: Track 1, transcribed and labelled RNA from the T7EBSP6 construct (Elenbaas consensus sequence) reacted with FL-MDM2; 2, FL-MDM2 protein post digestion with proteinase $\mathrm{K} ; 3$, mock reacted with Tris/ $\mathrm{HCl} ; 4$, randomised RNA sequence reacted with FL-MDM2 protein; 5, FL-MDM2 protein post digestion with proteinase $\mathrm{K} ; 6$, N50 random 50 mer transcript control.

Enzyme linked immunosorbent assay (ELISA). ELISAs were performed in accordance with, or as modifications of our conventional protocols (22). These various adaptations involved the use of different ligands as capture antigens, or capture antibodies. Thus variations were employed which allowed: i) determination of antibody concentrations with reference to standard sub-type specific titration curves. ii) Assessment of interaction of monoclonal antibodies with MDM2 or MDM2:nucleic acid complexes. Although the nature of the capture antibody immobilised on the solid phase of the plate varied in each of these assays, the same alkaline phosphatase conjugated rabbit

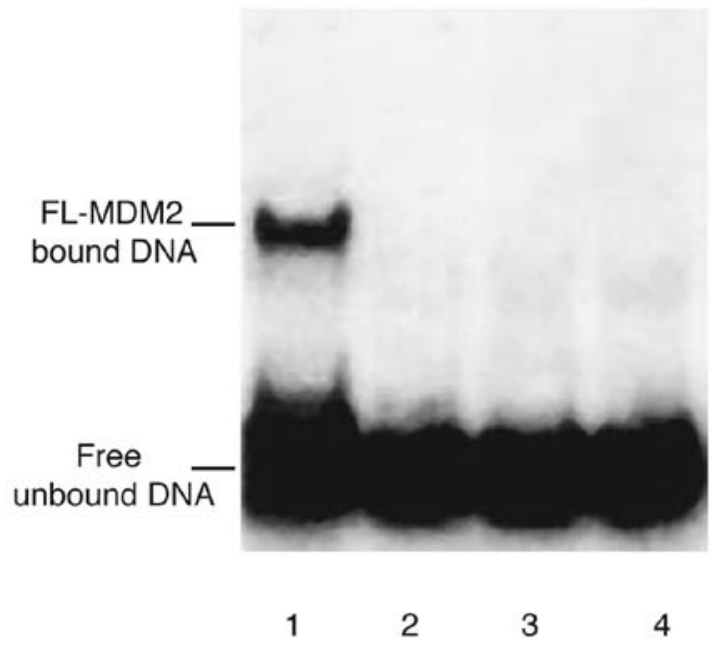

Figure 3. Binding of MDM2 selected DNA sequences (7th round selected sequences) to MDM2 in comparison to binding to control protein preparations. Features shown are: Track 1, MDM2 selected binding sequences reacting with FL-MDM2 protein; 2, with protein from lysates of bacteria transfected with pET $15 \mathrm{~b}$ alone; 3 , with protein from lysates of bacteria transfected with $\mathrm{pET}$ 15b-cyclin $\mathrm{D} 1 ; 4$, with Tris- $\mathrm{HCl}$ (no protein control).

anti-mouse immunoglobulin (KPL Laboratories) was employed $(1 / 10000)$ throughout. The chromagenic substrate employed in all experiments was p-nitro-phenol-phosphate (PNP) in alkaline phosphatase buffer. Reactions were halted with sodium hydroxide and binding of the conjugate to each target complex was visualised by cleavage of PNP, monitored by absorbance measurement at $590 \mathrm{~nm}$.

\section{Results}

Purification of MBP-MDM and FL-MDM2 proteins. MBPMDM2 and FL-MDM2 recombinant protein products were each successfully extracted using the described protocols. The purity of the final samples was established by a combination of PAGE and Western blotting using F2-2 and F4-14 antibodies (Fig. 1) (20).

RNA binding of the poly-histidine tagged full length MDM2 protein. Previous studies have shown that bacculovirus expressed MDM2 binds both L5/5S ribosomal ribo-nucleoprotein particles and specific RNA sequences or structures via the RING Finger domain. This subsequently led to the identification of an MDM2 RNA binding consensus sequence (16). To confirm our bacterial FL-MDM2 displayed similar RNA binding activity, an oligo-nucleotide containing the 30 mer RNA consensus sequence flanked by $\mathrm{Sp} 6$ and $\mathrm{T} 7$ promoter sequences (T7EBSp6) was synthesised and labelled alongside the control population of random N50 RNA (16). Binding and gel retardation assays show that the bacterial FL-MDM2 protein bound the T7EBSp6 consensus sequence (Fig. 2; lane 1). RNA binding was dependent upon the presence of the protein. Omission of protein or pre-digestion of protein with Proteinase $\mathrm{K}$ prior to binding completely abrogated specific RNA mobility shifts (Fig. 2, lanes 2 and 3). Furthermore, binding was correlated with protein concentration (data not included). 


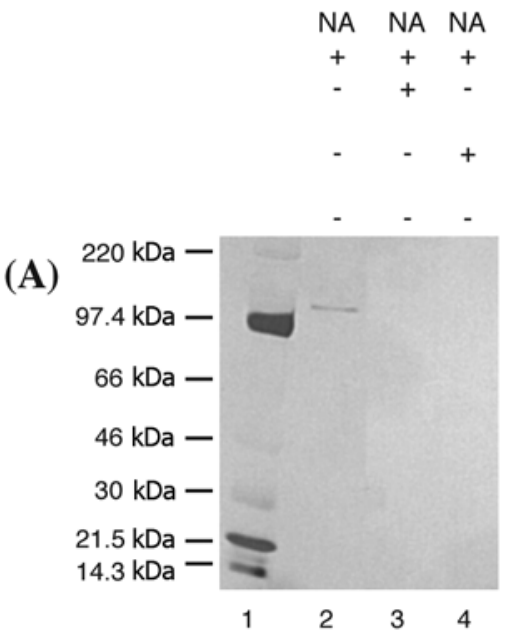

Silver stained polyacrylamide gel demonstrating immuno-absorbtion of MDM2 recombinant protein preparations

FL MDM2

\section{Clone 7B3 DNA}

Immuno-absorbed with bead immobilised anti-MDM2 MAbs (F2-2 \& F4-14)

Immuno-absorbed with bead immobilised anti-poly-histidine MAb

Mock Immuno-absorbtion (beads alone)

(B)

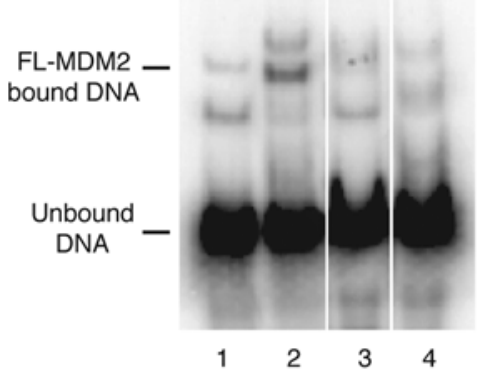

Mobility shift assay demonstrating the effect of immuno-absorbtion of MDM2 protein upon DNA binding

Figure 4. Depletion of specific MDM2 DNA binding capacity, with different immunoabsorbants. Features shown are (A) Track 1, molecular weight markers; 2 , recombinant poly-His tagged FL-MDM2 protein; 3, Poly-His tagged FL-MDM2 protein post immuno-absorption with immobilized anti-MDM2 monoclonal antibodies F2-2 and F4-14; Track 4, Poly-his tagged FL-MDM2 protein post immuno-absorption with immobilized anti-poly-histidine monoclonal antibody. (B) Track 1, DNA binding clone 7B3 reacted with Tris/HCl; 2, with FL-MDM2 protein; 3, with FL-MDM2 protein post-absorption with immuno-absorbent beads coated with anti-poly histidine monoclonal antibody; 4, with Tris/HCl itself mock absorbed with anti-MDM2 (F2-2 and F4-14) coated immuno-absorbent beads .

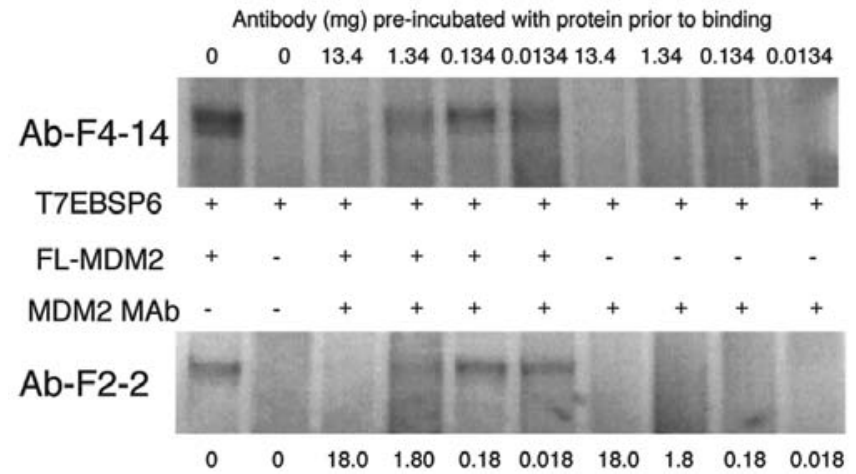

Figure 5. Inhibition of MDM2 RNA binding, by serial dilutions of anti-MDM2 monoclonal antibodies F4-14 and F2-2. Features shown are in vitro transcribed and ${ }^{32} \mathrm{P}-\mathrm{UTP}$ labelled RNA from the T7EBSP6 construct, reacted with $0.5 \mu \mathrm{g}$ FL-MDM2 protein following pre-incubation with serial dilutions of anti MDM2 antibodies F4-14 (upper gel) or F2-2 (lower gel).

A degree of protein binding was evident when FL-MDM2 protein was reacted with the random 50 mer (Fig. 2, lane 4). However, this protein dependent binding (lanes 5 and 6), was quite distinct from the specific binding between FL-MDM 2 and the T7EBSp6 consensus sequence and may reflect a degree of non-specific RNA:protein interaction.

DNA binding of the maltose binding protein (MBP)-MDM2 chimera. While the C-terminal region of MDM2 with its Ring Finger regions has been shown to bind RNA (16), its potential DNA binding properties have not been established. We found that the MBP-MDM2-(aa 708-1473) chimera bound a subset of the random radiolabelled DNA sequences from the primary R76

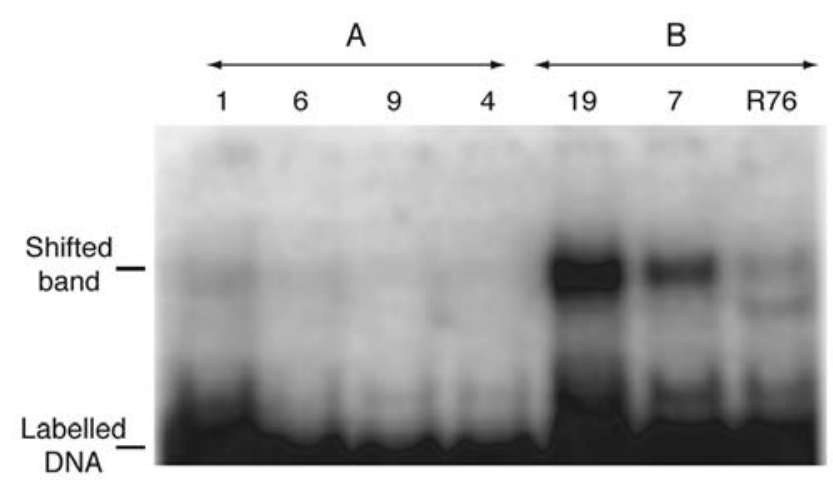

Figure 6. Cloned DNA sequences showing differential binding to recombinant FL-MDM2 protein, after A, initial selection (7th rounds); B, secondary selection (7+10); R76, random pool.

preparation. Sequences from the original random $\mathrm{R} 76$ sequences were enriched for MDM2 binding by successive rounds of MBP-MDM2 binding amylose column chromatography and PCR amplification. This process yielded a subpopulation of DNA sequences enriched on the basis of their MDM2 binding capacity (7th round selected). The increased specificity of the observed oligonucleotide/MDM2 binding was indicated by the reduced ability of progressively higher concentrations of poly-dIdC to inhibit binding of the unselected R76 sequences compared to little or no effect with the same concentrations on the selected sequences (data not shown). The DNA binding to the MBP chimera was protein dependent and not due to the MBP component. 
Table I. Comparative alignment of DNA binding sequences.

\begin{tabular}{lllc}
\hline Sequence & \multicolumn{1}{c}{ 5'-Nucleotide-3' $^{\prime}$} & U/Y & Binding affinity \\
\hline Consensus sequence & ATAAAGAACCAAGATTAGTAAAGGGGX & $21 / 26$ & \\
Empirical sequence & UYUUUUUUYYUUUUYYUUYUUUUUUU & $21 / 26$ & Strong \\
3BCON & GAAAAGAACCAAGAGGTGTATGTGGG & $19 / 26$ & Strong \\
19A & GGTAAATACAAGAGTAACTAGAGGTG & $19 / 26$ & Strong \\
$7 \mathrm{~B} 3$ & TTCAATTATTGTACTCAAGGAGAGAG & $15 / 26$ & Strong \\
$29 \mathrm{BF}$ & AGACGAAACAAAACGTAACCAGTTGG & $19 / 26$ & Strong \\
$28 \mathrm{BF}$ & ATAAAGGCTGAAGTACTGGAGAGGGGG & $21 / 27$ & Weak \\
$20 \mathrm{~A} 1$ & AGAAAGAGCGGTATAGAGCTAGGCAA & $20 / 26$ & Weak \\
$9 \mathrm{BF}$ & ACTGTGGTTCTTGTATACGTTACGAT & $11 / 26$ & Weak \\
$6 \mathrm{BF}$ & GTTTGAAGACTGTACAGGAGACTGGA & $17 / 26$ & Weak \\
$1 \mathrm{BF}$ & GCAGGTGCGAAAGAGTTGTATAGGTG & $18 / 26$ & Weak \\
$17 \mathrm{BF}$ & ACAATACCATTAGATGGACAGAGCAA & $17 / 26$ & \\
\hline
\end{tabular}

DNA binding of the poly-histidine tagged full length MDM2 protein. In the mobility shift assays with the MBP-MDM2 chimera it was observed that the bound complexes displayed very low mobility, being retarded in the extreme upper (well) portion of the gel. This very low mobility appeared to be related to the high molecular weight of the MBP chimera. A full-length poly-histidine tagged MDM2 protein was therefore used instead of this chimera. This protein, like the MBP-MDM2 chimera, showed significant DNA binding. Further rounds of extraction and amplification, as described, led to the isolation of sequences exhibiting even greater binding efficiency to FL MDM2 protein (7+10 selected DNA). This binding again was protein dependent and unrelated to the presence of the poly-histidine tag. Control protein preparations including protein extracts from lysates of IPTG induced broth cultures of E. coli BL21-DE3 transformed with (a) the pET $15 \mathrm{~b}$ vector alone or (b) $\mathrm{pET} 15 \mathrm{~b}$ vector containing the coding sequence of a protein unrelated to MDM2, in this instance cyclin D1. In DNA binding/mobility shift assays, in contrast to the FL-MDM2 protein, both control lysates failed to bind the selected DNA (Fig. 3).

Finally, immuno-absorption was used to further confirm that the observed DNA binding was MDM2 specific. Initially both absorbed and pre-absorbed protein samples were resolved by SDS-PAGE and silver staining confirmed the efficacy of immune-absorption (Fig. 4A). These fractions were then used in corresponding DNA binding reactions. Specific immunoabsorption of MDM2 (Fig. 4A, tracks 3 and 4) corresponded with failure to bind selected DNA (Fig. 4B, tracks 3 and 4).

Comparison of DNA and RNA binding. The bacterially expressed FL-MDM2 protein displayed both DNA and RNA binding activities. A series of blocking experiments were performed employing monoclonal antibodies in order to further affirm the protein specificity of each of the protein:nucleic acid interactions observed.

Cloning and sequencing of the DNA oligonucleotide binding $M D M 2$. To determine if there was a DNA binding consensus sequence or any defined regular sequence patterns were present in the population of enriched MDM2 DNA binding sequences, these as well as control unselected random pool R76 sequences were amplified and cloned into pCR3.1 as described. The sequences corresponding to the inserts within these clones were subsequently amplified radiolabelled and their binding to FL-MDM2 was evaluated in mobility shift assays. Comparison of the results of these binding assays showed that these cloned sequences possessed a range of different binding affinities for MDM2 (Fig. 6). Selected DNA clones, with inserts which when amplified by PCR exhibited a range of MDM2 binding activities, were sequenced and the results comparatively aligned using Clustal W, Blast and DNASTAR software. Among the high affinity sequences analysed regular runs of polypurines:polypyrimidines were evident throughout (21/26 purines), although complete sequence concordance was not observed (Table I). While no classic DNA binding consensus sequence was observed, polypurine runs (polypyrimidine on the complementary strands) appeared to be generally selected for and particularly characteristic of clones with high binding affinity.

\section{Discussion}

Although attention has mainly focused on the function of MDM2 as a negative regulator of the p53 tumour suppressor, the structure of the C-terminus of MDM2, which includes acidic, Zinc Finger and Zinc RING Finger domains, suggests that the human onco-protein MDM2 may possess nucleic acid binding or transcription factor function. It has been found that bacculovirus expressed MDM2 protein 'binds the L5/5S ribosomal ribo-nucleotide particle and can also bind specific RNA sequences and structures'. These interactions appear to be mediated through the RING Finger Domain of the protein, and has led to the identification of a consensus RNA sequence (16).

To date DNA binding properties of the MDM2 protein have not been described. The present study investigated and compared the ability of bacterially expressed MDM2 protein to bind both the reported consensus RNA sequence and explored its capacity to bind DNA. Cloning and expression of partial 
(MBP-MDM2) and full length MDM2 (FL-MDM2) cDNAs, together with the subsequent purification procedures, provided proteins for these binding studies.

Purified FL-MDM2 protein bound avidly in a sequence, and protein-dependent manner to the previously reported T7EBSp6 RNA consensus sequence (16). Although a degree of RNA binding was evident when this protein was reacted with the random 50 mer, this appeared distinct in character, more consistent with a non-specific RNA:protein interaction. RNA binding inferred conformational integrity of the FL-MDM2 preparation, which in other studies we have also shown to bind p53. DNA binding studies employed both an MBP-MDM2-C-Terminal chimera as well as the poly-histidine tagged FL-MDM2 protein to select reactive DNA sequences. Binding of both the MBP-MDM2 and MBP-MDM2/ FL-MDM2 selected sequences proved robust and specific, as complex formation was observed in the presence of high levels of poly-dIdC competitor. The dependence on protein integrity and conformation for both MDM2:DNA and MDM2:RNA complex formation was demonstrated by its sensitivity to Proteinase K digestion and heat denaturation. DNA binding was also shown to be independent of either the MBP or polyhistidine tags or PCR reaction components. No binding was evident in control reactions, which included: MBP alone, or proteins prepared from lysates of cells transfected with pET15b alone or a pET15b-cyclin D1 expression construct (a protein known not to possess significant DNA binding activity). Additionally, immunoabsorption of FL-MDM2 with MDM2 specific monoclonal antibodies linked to Sepharose was found to eliminate the DNA and RNA binding activity of MDM2 protein preparations.

RNA and DNA binding, respectively, may involve different domains of the protein. Antibodies F2-2 and F4-14 (N-terminal reactive) inhibited RNA binding, although to different degrees. Epitope mapping using short random fragments of MDM2 (40-50 nucleotides), expressed utilising the pScreen vector/E. coli system, indicated that antibody F2-2 recognised what appeared to be a discrete amino acid sequence between amino acids 210 and 235 (core comprising amino acids 220 to 229). In contrast, antibody F4-14 appeared to recognise a discontinuous epitope with clusters of contact residues present between amino acids 146 and 160 as well as 212 and 224. Thus no contact residues of either antibody Complementary Determining Region (CDR) lie within the RING Finger Domain mapping between amino acids 238 and 278. Inhibition is most likely to be a result of steric hindrance rather than direct competition between antibody and RNA consensus sequence for contact residues. In contrast to the effect upon RNA binding, additional ELISA experiments suggested that neither antibody interfered with DNA binding, and both antibodies retained their ability to bind to MDM2 when it was complexed to DNA. Together these observations suggest that MDM2:DNA interaction occurs at site(s) away from the NLSs, $\mathrm{N}$ terminal portion of the Acidic Activation Domain and the RING Finger Motif.

On cloning and sequencing the selected DNA PCR products that bound MDM2, a heterogeneous series of sequences were identified, which displayed varying binding affinities for FL-MDM2. All cloned sequences, including high and low affinity clones, were comparatively aligned using BLAST and Clustal W software. All clones possessed high proportions of polypurine/polypyrimidine tracts. However, while polypurine runs were particularly characteristic of clones with high binding affinity, no complete sequence concordance or consensus binding sequence was evident amongst them. These findings contrast starkly to those obtained in reported studies of RNA binding where high sequence homology was apparent among MDM2 binding RNA clones with differences being observed in only 2-5 nucleotides (16).

Protein:nucleic acid and protein:protein interactions are known to be involved in many cellular processes including: transcription, regulation of cellular differentiation, transformation and ubiquitination in protein degradative pathways. These protein interactions are facilitated by a number of specialised structures including classic Zinc Finger motifs as well as the more recently characterised $\mathrm{C}_{3} \mathrm{HC}_{4}$ zinc binding domain or RING Finger motif which takes its name from the gene in which it was first identified namely the human RING 1 gene (23). Classic Zinc Fingers were first described in the Xenopus TFIIIA transcription factor, in which nine such motifs were observed in tandem (24). These motifs were subsequently found to function both as specific RNA and DNA binding modules with recognition occurring by means of distinct mechanisms. This is particularly intriguing, as we demonstrate RNA binding through the RING Finger motif and cannot exclude the possibility of the involvement of the classic Zinc Finger or the RING Finger in MDM2:DNA binding. RING Finger Domains have been identified in a number of proteins with oncogenic potential $(25,26)$. These proteins include the PML onco-protein, the bacculovirus inhibitor of apoptosis IAP proteins (27), Mel 18 associated with murine melanoma (8) and finally the breast cancer susceptibility gene BRCA-1 (28). While it has been shown that RING Finger domains can bind to synthetic DNA oligonucleotides and/or DNA cellulose in a zinc dependent manner $(23,29)$ sequence specific examples have not been described. Thus if the DNA binding observed in our studies does involve Zinc or RING Finger Domains, the absence of a specific consensus DNA binding sequence is consistent with the above observations. It is possible that binding may occur as a result of charge interactions through the positively charged RING Finger.

As mentioned previously, the RING Finger domain of the Mel 18 protein has been found to bind oligonucleotides in a sequence-independent manner. However, studies have also shown that the Mel 18 protein can also bind DNA in a sequence dependent manner, although the RING Finger appears not to be implicated (26). Although we found no highly specific DNA consensus recognition sequence, the occurrence of a high proportion of regular runs of purine/ pyrimidine was apparent in the MDM2 binding clones. This suggests that binding involves a degree of selective binding to polypurine/pyrimidine tracts of DNA, perhaps reflecting a specific functional role. This observation complements that made previously, which demonstrated that MDM2 would bind and co-precipitate homo-ribopolymer-G, i.e. a homo-ribopyrimidine (16). Direct comparison of the results of previous research and the present study must be performed with caution, as much previous work has relied upon immuno-precipitation studies, rather than direct binding assays such as the mobility shift assays and selection used in the present study. The results of immuno-precipitations rely on the reactivity of the antibody 
with the protein:nucleic acid complex as well as the native protein.

Homopurine/pyrimidine tracts have been identified in the promoter regions of a number of distinct genes that are often associated with growth control functions. One such region has been identified in the promoter of the PDGF $\alpha$ chain (30). Other examples include Myc, and Myc-N. Thus it is conceivable that motifs similar to that observed in the present study may exist in promoters of other genes. MDM2 binding to such promoter sequences may allow modulation of downstream gene expression.

The research presented in the present report did not seek to define the amino acids on MDM2 that binds to nucleic acid. However, previous research shows RNA binding to occur through the RING Finger and our present research shows that antibodies F4-14 and F2-2 may sterically inhibit this binding to RNA. These same antibodies did not interfere with DNA binding, implying that this binding does not involve the RING Finger, although this possibility cannot be completely excluded. Evidence in the literature indicates Zinc Finger motifs to be associated with DNA binding. Thus, MDM2 may show some similarities to binding of DNA by the Xenopus TFIIIa protein where classic Zinc Fingers are involved in DNA binding as well as RNA binding. The MDM2 protein contains tandem carboxy proximal Zinc Fingers of the $\mathrm{C} 2 \mathrm{H} 2$ and $\mathrm{C} 4$ classes and the possibility remains that these sequences may be involved in the observed DNA binding. This hypothesis and the mapping of the MDM2 protein:DNA binding sequences would be of interest to investigate further. Increasing numbers of research papers suggest that RING Finger motifs, in contrast to the classic Zinc Fingers, are more often involved in protein:protein interactions. Examples include the BRCA-1 gene that possesses a RING Finger domain, which binds the BARD-1 and BAP1 proteins $(31,32)$. Such proteins are known to exhibit a number of ubiquitin-dependent regulatory activities in their own right including: transcription, chromatin re-modelling, cell cycle control and DNA repair. The MDM2 protein has been shown to fulfil a role in ubiquitin-dependent regulatory processes and acts as a zinc-dependent E3 protein ligase involved in p53 degradation (33), but it is also regulated through the RING Finger Domain $(19,34)$. p14 ${ }^{\mathrm{ARF}}$ and MDMX both bind to the MDM2 RING Finger. While p14 ${ }^{\mathrm{ARF}}$ inhibits MDM2 ubiquitindependent ligase activity so inhibiting p53 degradation, MDMX inhibits ubiquitin-dependent proteosome-dependent degradation of MDM2 itself. In a mutational analysis of the Ring finger domain of MDM2 Singh et al have found that MDMX can efficiently rescue both ubiquitin and Nedd8 ligase activity of these ring finger mutants (35). Since the interaction between MDM2 and MDMX is more stable than MDM2 homo-dimerisation this suggests that MDM2-MDMX complexes play a prominent role in 553 ubiquitination in vivo. Additional studies by Giglio et al have shown that the oncogenic isoform of MDMX, MDMX11 binds and stabilises MDM2 (36). Although MDMX11 lacks the p53-binding site it stabilises p53 by counteracting its degradation by MDM2. These observations confirm the importance of the role of both the homomeric MDM2-MDM2 complexes as well as the heteromeric MDM2-MDMX, and the interactions with MDM2 and isoforms of MDMX, in the ubiquitination mediated p53 degradation pathways. In addition, Linares et al have found that the presence of nucleic acids (homo-polymeric poly-riboncleotides and poly-deoxyriboncleotides) inhibits both the E3 activity of MDM2 and the ability to form homodimeric complexes, providing a novel mechanism by which MDM2 mediated ubiquitination processes can be regulated (37). While they found no difference between efficiency of DNA and RNA homo-polymers in their ability to inhibit E3 activity, the consensus RNA binding sequence may well be more physiologically significant than initially thought. Our identification of clones of DNA sequences with differential binding to MDM2 begs the question as to their possible role in the modulation of ubiquitination, along with the Elenbaas consensus RNA binding sequence. Future research into modulation of ubiquitination by these nucleic acids may provide useful information for the design and use of therapeutic approaches directed against the function of MDM2.

MDM2 is also regulated by ribosomal proteins, which bind to the central acidic domain and inhibit its E3 ubiquitin ligase activity. Lindstrom et al have recently shown that cancer associated mutations in the central zinc finger domain disrupt the interaction of MDM2 with the ribosomal proteins L5 and L11 and attenuate MDM2 induced p53 degradation, illustrating the importance of both the Zinc and Ring finger structures in the ubiquitination, MDM2/p53 degradation pathways (38).

The bacterially expressed human MDM2 protein investigated in this study showed selective binding to DNA sequences rich in polypurine/pyrimidine tracts. Research on a wide range of DNA binding proteins has shown that DNA binding may be regulated/modulated by phosphorylation $(39,40)$. MDM2 possesses a number of consensus phosphorylation sites, the majority of which are serine-containing sites, although threonine and tyrosine sites are also present. A number of these sites reside in the Classic Zinc Finger as well as the RING Finger regions. It would therefore be of interest to explore the potential effect that phosphorylation/dephosphorylation of these sites within different sub-cellular compartments may exert upon MDM2 binding of both DNA and RNA.

In summary, we have shown that a bacterially expressed MDM2 protein binds both RNA and DNA. This result supports the finding that MDM2 binds RNA and that this interaction occurs in a sequence-dependent manner involving the RING Finger Domain. Furthermore, we have demonstrated the ability of MDM2 to bind DNA. However, the mechanism of DNA binding appears to be distinct from that of RNA binding. Although we may speculate upon the potential sites upon the protein that may be involved in this binding, the present report does not definitively identify this/these region(s). Further research will be required to define the precise protein residues involved.

\section{Acknowledgements}

Support for this research was provided by Cancer Research UK. We would also like to thank Professor Andrew Sharrocks who kindly supplied the R76 PF and PR primers used in this study, and Dr Stephen Crosier for his aid and critique.

\section{References}

1. Barak Y, Juven T, Haffner R and Oren M: mdm2 expression is induced by wild type p53 activity. EMBO J 12: 461-468, 1993. 
2. Farmer G, Bargonetti J, Zhu H, Friedman P, Prywes R and Prives C: Wild-type p53 activates transcription in vitro. Nature 358: 83-86, 1992.

3. Fakharzadeh SS, Trusko SP and George Dl: Tumorigenic potential associated with enhanced expression of a gene that is amplified in a mouse tumor cell line. EMBO J 10: 1565-1569, 1991.

4. Picksley SM and Lane DP: The p53-mdm2 autoregulatory feedback loop: a paradigm for the regulation of growth control by p53? Bioessays 15: 689-690, 1993.

5. Wu XW and Bayle JH: The p53 mdm-2 autoregulatory feedback loop. Genes Dev 7: 1126-1132, 1993.

6. Honda R, Tanaka H and Yasuda H: Oncoprotein MDM2 is a ubiquitin ligase E3 for tumor suppressor p53. FEBS Lett 420 25-27, 1997.

7. Momand J,Zambetti GP, Olson DC, George D and Levine AJ: The mdm-2 oncogene product forms a complex with the p53 protein and inhibits p53-mediated transactivation. Cell 69: 1237-1245, 1992.

8. Oliner JD, Pietenpol JA, Thiagalingam S, Gyuris J, Kinzler KW and Vogelstein B: Oncoprotein MDM2 conceals the activation domain of tumour suppressor p53. Nature 362: 857-860, 1993.

9. Sigalas I, Calvert AH, Anderson JJ, Neal DE and Lunec J: Alternatively spliced mdm 2 transcripts with loss of p53 binding domain sequences: transforming ability and frequent detection in human cancer. Nat Med 2: 912-917, 1996.

10. Xiao ZX, Chen J, Levine AJ, Modjtahedi N, Xing J, Sellers WR and Livingston DM: Interaction between the retinoblastoma protein and the oncoprotein MDM2. Nature 375: 694-698, 1995.

11. Martin K, Trouche D, Hagemeier C, Sorensen TS, La Thangue NB and Kouzarides T: Stimulation of E2F1/DP1 transcriptional activity by MDM2 oncoprotein. Nature 375: 691-694, 1995.

12. Marechal V, Elenbaas B, Piette J, Nicolas JC and Levine AJ: The ribosomal L5 protein is associated with mdm-2 and mdm-2-p53 complexes. Mol Cell Biol 14: 7414-7420, 1994.

13. Dobbelstein M, Wienzek S, Konig C and Roth J: Inactivation of the p53-homologue p73 by the mdm2-oncoprotein. Oncogene 18: 2101-2106, 1999

14. Boddy MN, Freemont PS and Borden Kl: The p53-associated protein MDM2 contains a newly characterized zinc-binding domain called the RING finger. Trends Biochem Sci 19: 198-199, 1994.

15. Chen J, Marechal V and Levine A: Mapping of the p53 and mdm-2 interaction domains. Mol Cell Biol 13: 4107-4114, 1993.

16. Elenbaas B, Dobbelstein M, Roth J, Shenk T and Levine AJ: The MDM2 oncoprotein binds specifically to RNA through its RING finger domain. Mol Med 2: 439-451, 1996.

17. Freemont PS: RING for destruction? Curr Biol 10: R84-R87, 2000

18. Stott FJ, Bates S, James MC, McConnell BB, Starborg M, Brookes S, Palmero I, et al: The alternative product from the human CDKN2A locus, p14(ARF), participates in a regulatory feedback loop with p53 and MDM2. EMBO J 17: 5001-5014, 1998

19. Tanimura S, Ohtsuka S, Mitsui K, Shirouzu K, Yoshimura A and Ohtsubo M: MDM2 interacts with MDMX through their RING finger domains. FEBS Lett 447: 5-9, 1999.

20. Anderson JJ, Challen C, Atkins H, Suaeyun R, Crosier S and Lunec J: MDM2 RNA binding is blocked by novel monoclonal antibody h-MDM2-F4-14. Int J Oncol 31: 45-56 2007.

21. Pollock R and Treisman R: A sensitive method for the determination of protein-DNA binding specificities. Nucleic Acids Res 18 6197-6204, 1990.

22. Mcintosh GG, Anderson JJ, Milton I, Steward M, Parr AH, Thomas MD, Henry JA, et al: Determination of the prognostic value of cyclin D1 overexpression in breast cancer. Oncogene 11: 885-891, 1995
23. Lovering R, Hanson IM, Borden KL, Martin S, O'Reilly NJ, Evan GI, Rahman D, et al: Identification and preliminary characterization of a protein motif related to the zinc finger. Proc Natl Acad Sci USA 90: 2112-2116, 1993

24. Theunissen O, Rudt F, Guddat U, Mentzel H and Pieler T: RNA and DNA binding zinc fingers in Xenopus TFIIIA. Cell 71: 679-690, 1992.

25. Freemont PS: The RING finger. A novel protein sequence motif related to the zinc finger. Ann N Y Acad Sci 684: 174-192, 1993.

26. Saurin AJ, Borden KL, Boddy MN and Freemont PS: Does this have a familiar RING? Trends Biochem Sci 21: 208-214, 1996.

27. Miller LK: An exegesis of IAPs: salvation and surprises from BIR motifs. Trends Cell Biol 9: 323-328, 1999.

28. Miki Y, Swensen J, Shattuck-Eidens D, Futreal PA, Harshman K, Tavtigian S, Liu Q, et al: A strong candidate for the breast and ovarian cancer susceptibility gene BRCA1. Science 266: 66-71, 1994.

29. Takahashi M, Inaguma Y, Hiai H and Hirose F: Developmentally regulated expression of a human 'finger'-containing gene encoded by the $5^{\prime}$ half of the ret transforming gene. Mol Cell Biol 8: $1853-1856,1988$.

30. Wang ZY and Deuel TF: An S1 nuclease-sensitive homopurine/ homopyrimidine domain in the PDGF A-chain promoter contains a novel binding site for the growth factor-inducible protein EGR-1. Biochem Biophys Res Commun 188: 433-439, 1992.

31. Jensen DE, Proctor M, Marquis ST, Gardner HP, Ha SI, Chodosh LA, Ishov AM, et al: BAP1: a novel ubiquitin hydrolase which binds to the BRCA1 RING finger and enhances BRCA1mediated cell growth suppression. Oncogene 16: 1097-1112, 1998.

32. Wu LC, Wang ZW, Tsan JT, Spillman MA, Phung A, Xu XL, Yang MC, et al: Identification of a RING protein that can interact in vivo with the BRCA1 gene product. Nat Genet 14: 430-440, 1996.

33. Fang S, Jensen JP, Ludwig RL, Vousden KH and Weissman AM: $\mathrm{Mdm} 2$ is a RING finger-dependent ubiquitin protein ligase for itself and p53. J Biol Chem 275: 8945-8951, 2000.

34. Honda R and Yasuda H: Association of p19 (ARF) with Mdm2 inhibits ubiquitin ligase activity of $\mathrm{Mdm} 2$ for tumor suppressor p53. EMBO J 18: 22-27, 1999.

35. Singh RK, Iyappan S and Scheffner M: Hetero-oligomerization with MdmX rescues the ubiquitin/Nedd8 ligase activity of RING finger mutants of Mdm2. J Biol Chem 282: 10901-10907, 2007.

36. Giglio S, Mancini F, Gentiletti F, Sparaco G, Felicioni L, Barassi F, Martella C, et al: Identification of an aberrantly spliced form of HDMX in human tumors: a new mechanism for HDM2 stabilization. Cancer Res 65: 9687-9694, 2005.

37. Linares LK and Scheffner M: The ubiquitin-protein ligase activity of $\mathrm{Hdm} 2$ is inhibited by nucleic acids. FEBS Lett 554: 73-76, 2003.

38. Lindstrom MS, Jin A, Deisenroth C, White Wolf G and Zhang Y: Cancer-associated mutations in the MDM2 zinc finger domain disrupt ribosomal protein interaction and attenuate MDM2-induced p53 degradation. Mol Cell Biol 27: 1056-1068, 2007.

39. Dang J, Kuo ML, Eischen CM, Stepanova L, Sherr CJ and Roussel MF: The RING domain of Mdm2 can inhibit cell proliferation. Cancer Res 62: 1222-1230, 2002.

40. Martinez JD, Craven MT, Joseloff E, Milczarek G and Bowden GT: Regulation of DNA binding and transactivation in p53 by nuclear localization and phosphorylation. Oncogene 14: 2511-2520, 1997. 